\title{
The PDF interpretation of the measurement of a $W$ boson produced in association with jets at the ATLAS detector
}

\section{Mark Sutton ${ }^{* \dagger}$}

Department of Physics, The University of Sussex, Brighton, UK

E-mail: suttecern.ch

\begin{abstract}
A QCD analysis using heavy vector boson data from the ATLAS detector at the LHC collected during the 2012 running period at a centre-of-mass energy of $8 \mathrm{TeV}$, where the boson was produced in association with at least one hadronic jet, is presented. Included in the analysis are ATLAS data on inclusive $W$ and $Z$ boson production at $7 \mathrm{TeV}$. The results are presented together with a new fit to the ATLAS inclusive $W$ and $Z$ data alone, performed with an updated parameterisation. The consequences for the strangeness content of the proton are discussed. Systematic correlations between the input ATLAS data sets are fully accounted. The resulting PDF set is called ATLASepWZWjets19.
\end{abstract}

XXVII International Workshop on Deep-Inelastic Scattering and Related Subjects - DIS2019 8-12 April, 2019

Torino, Italy

* Speaker.

${ }^{\dagger}$ On behalf of the ATLAS Collaboration 


\section{Introduction}

The LHC [1] is an excellent machine for the study of perturbative QCD. Essentially all hard processes at the LHC result from the interaction between partons - quarks or gluons - in the proton and precise knowledge of the parton densities is therefore an essential prerequisite for the detailed understanding of the Standard Model or identification of any possible signature of physics beyond the Standard Model.

During Run 1, the LHC performed extremely well, allowing the ATLAS collaboration [2] to collect a proton-proton data sample in excess of $20 \mathrm{fb}^{-1}$ at $8 \mathrm{TeV}$ centre-of-mass energy and in excess of $4 \mathrm{fb}^{-1}$ at $7 \mathrm{TeV}$. When performing a QCD analysis for the proton parton distributions, different physics processes provide information on the different parton initial states. The very precise HERA inclusive DIS data typically only tightly constrain the parton distributions at lower $x$, and are sensitive to the gluon at next-to-leading order (NLO) only through scaling violations. The LHC cross section on the other hand, with two target hadrons, is sensitive to the gluon distribution and the strong coupling already at leading order (LO) for $t \bar{t}$ production or processes including hadronic jets. Electroweak boson production is sensitive both to the valence and sea quark distributions, and where such a boson is accompanied by a jet in the final state, then these interactions are also directly sensitive to the gluon distribution. In this case, the additional emission typically results in enhanced sensitivity to higher $x$ than inclusive boson production.

For hadronic interaction processes, the cross section is typically only calculable after the numerical integration over the phase space to cancel the infrared divergences. As such, fast grid convolution techniques are required to reproduce the QCD calculation used in the fit. For this analysis such grids are generated using MCFM [3, 4] interfaced to APPLgrid [5, 6] with next-tonext-to-leading (NNLO) $K$-factors evaluated using DYNNLO [7, 8] and FEWZ [9, 10].

Previous QCD analyses $[11,12,13]$ which include the ATLAS inclusive vector boson production data provide evidence for an enhanced strangeness contribution at lower $x$.

\section{QCD analysis}

At the time of writing, ATLAS has produced several QCD analyses including ATLAS data on inclusive vector boson production: the epWZ12[11], and epWZ16 [11] fits, using $35 \mathrm{pb}^{-1}$ and $4.6 \mathrm{fb}^{-1}$ of inclusive $W$ and $Z$ boson production data at $7 \mathrm{TeV}$ respectively, and the epWZtop18 fit [13] which augments the epWZ16 fit by the inclusion of the leptonic [14] and semi-leptonic [15] ATLAS $t \bar{t}$ data.

The starting point for the current QCD analysis is the data used for the epWZ16 fit, with the addition of the ATLAS $W+$ jet data at $8 \mathrm{TeV}$ [16]. There are some differences with respect to the epWZ16 fit to accommodate or exploit the new data. A larger number of PDF parameter variations have been used as part of the model systematic uncertainties, together with an updated parameterisation with additional terms in the central fit for the $\bar{u}$ density, consistent with that used for the epWZtop18 analysis. This results in a 16 parameter fit with a starting scale of $Q^{2}=1.9 \mathrm{GeV}^{2}$. Altogether there were 131 sources of correlated systematic uncertainty in the inclusive data with the electron and muon channels combined. For the new analysis the electron and muon data have been used uncombined to allow a more straightforward correlation of the systematic uncertainties 

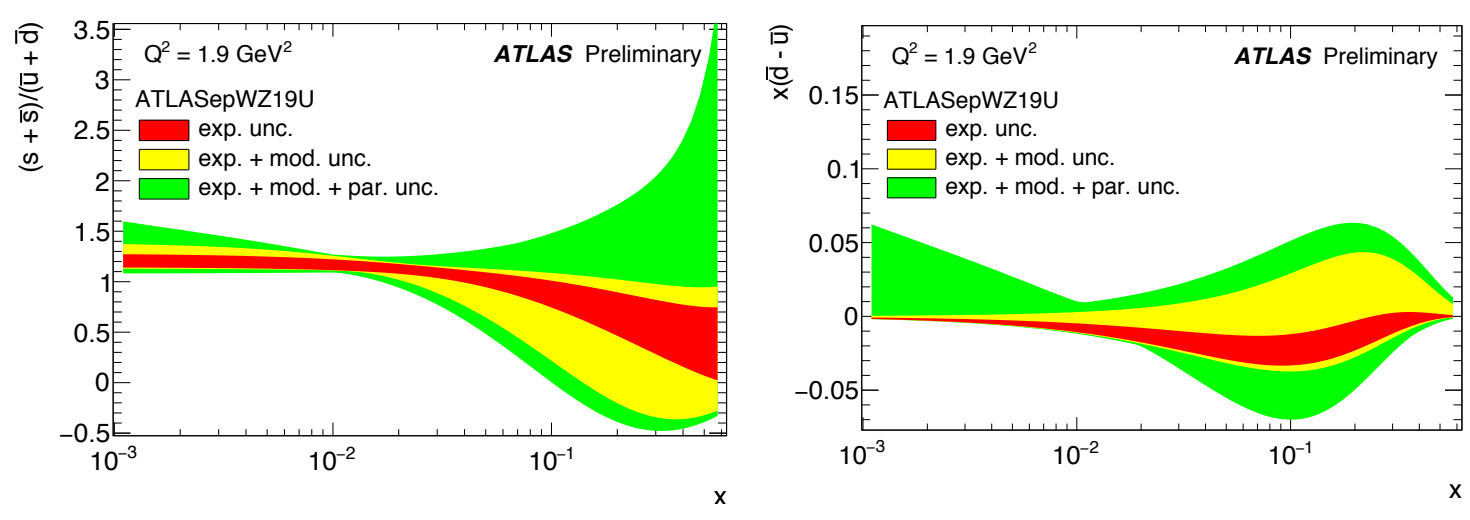

Figure 1: The strangeness ratio, $r_{s}=(s+\bar{s}) /(\bar{u}+\bar{d})$ (left) and the light quark asymmetry $x(\bar{d}-\bar{u})$ (right) for the new fit to the inclusive $W$ and $Z$ data. The inner error band shows the experimental uncertainty, the outer error bands include the model and parameterisation uncerainties.

with those from the $W+$ jet measurement. The new fits to the inclusive data are referred to as the ATLASepWZ19C, and ATLASepWZ19U fits for the fits using the combined, or uncombined data respectively, and the fit including the $W+$ jet data is referred to as ATLASepWZWjets19. In addition, to the new parameterisation used for the fit, the minimum $Q^{2}$ of the HERA DIS data used has been increased from $7.5 \mathrm{GeV}^{2}$ to $10 \mathrm{GeV}^{2}$ to better exclude the low- $x$, low $Q^{2}$ data that may be more adversely affected by higher twist and other effects.

Figure 1 shows the strangeness ratio $r_{s}=(s+\bar{s}) /(\bar{u}+\bar{d})$ and the light quark asymmetry, $x(\bar{d}-$ $\bar{u})$ for the new fits to the inclusive vector boson data only. As with the original ATLASepWZ16 fit, a negative value for the light quark asymmetry is observed with a negative peak around $x=10^{-1}$. Although negative, within the large model uncertainty this is still consistent with zero, and the large contribution from the strange density at low $x$ is reproduced.

The information on the full correlations between the different spectra $-p_{\mathrm{T}}(W)$, and $p_{\mathrm{T}}$ of the leading jet - for the $W+$ jet data is not available and as such it is not possible to include the data from both distributions simultaneously. Fits including each of the spectra individually, however, provide consistent results and give values for the $\chi^{2}$ per degree of freedom of 1354/1140 and 1365/1152 respectively for fits including the data for $p_{\mathrm{T}}(W)$, and $p_{\mathrm{T}}$ of the leading jet. The $p_{\mathrm{T}}(W)$ distribution is chosen for the central fit, with the distribution for the $p_{\mathrm{T}}$ of the leading jet fitted as a cross check. For the model variation uncertainties, several additional parameters are allowed to be free in the fit, but in all cases the $\chi^{2}$ per degree of freedom is consistent with the central fit.

Figure 2 compares the parton distributions from the new inclusive fit to the uncombined inclusive $W$ and $Z$ data with the new fit including the $W+$ jet data. The fits are consistent, but with the new fit including the $W+$ jet data favouring harder $\bar{d}$, and softer $d$ valence quark distributions, with the $u$ quark distributions largely unchanged. The uncertainty on the gluon density is largely unchanged, however, the gluon distribution itself in the fit with the $p_{\mathrm{T}}(W)$ is very slightly harder.

Looking in more details at the strangeness contributions in Figure 3 for the fit including the $W+$ jet data, the uncertainty at larger $x$ is very greatly reduced largely due to the increased sensitivity to larger $x$ from the additional radiation. The actual value of the $r_{s}$ ratio at large $x$ is reduced with respect to the fit to inclusive data only. However, the enhanced strangeness contribution at low $x$, 

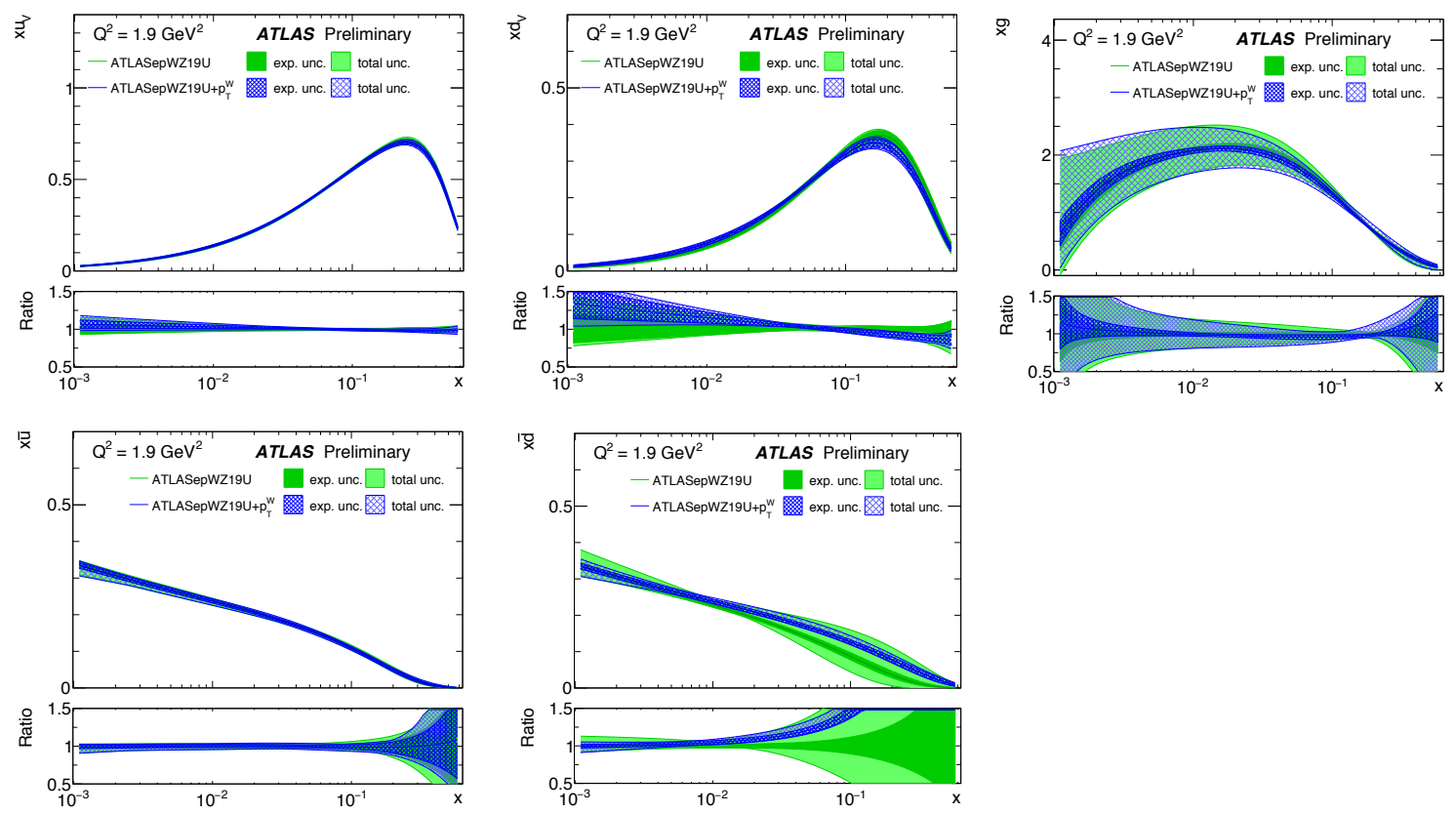

Figure 2: A comparison of the $u$ and $d$ quark, and gluon distributions for the new fit to the uncombined inclusive $W$ and $Z$ data with the new fit including also the data for the $p_{\mathrm{T}}(W)$ distribution.
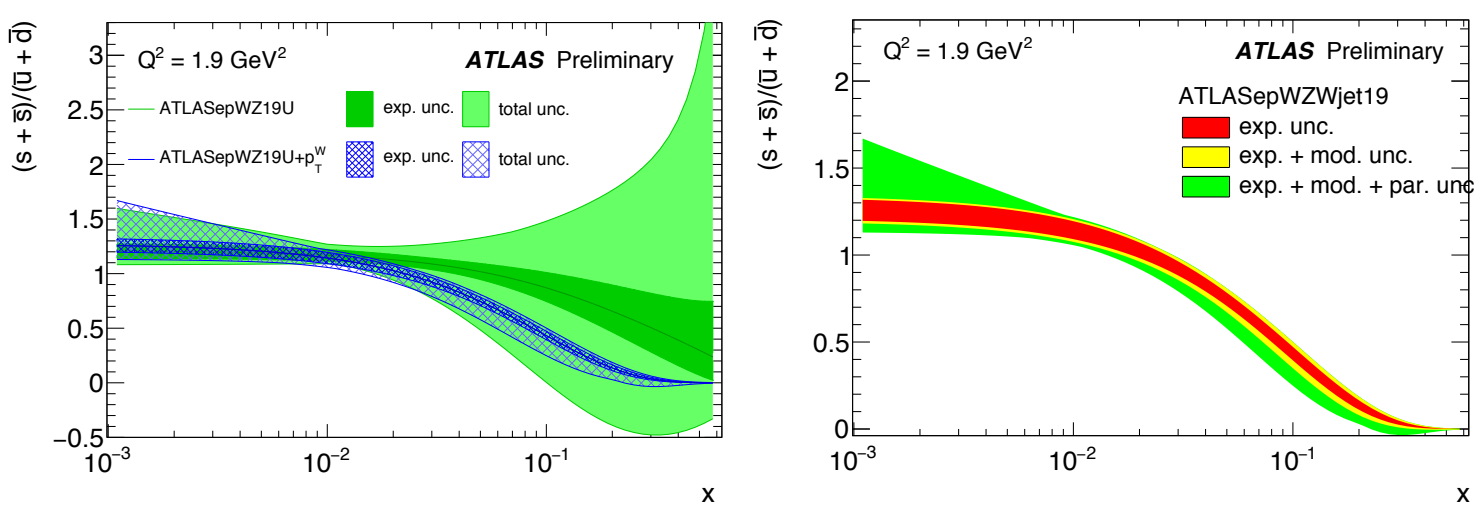

Figure 3: The strangeness ratio, $r_{s}=(s+\bar{s}) /(\bar{u}+\bar{d})$ for the new ATLASepWZ19U fit and the ATLASepWZWjets 19 fit including the $p_{\mathrm{T}}(W)$ data (left), and showing the breakdown of the uncertainties into experimental, model and parameterisation uncertainties (right).

which is again greater than unity, is still very much in evidence. Both the fits to the leading jet $p_{\mathrm{T}}$ distribution and to $p_{\mathrm{T}}(W)$ show a consistent behaviour and agree well within their respective uncertainties.

Figure 4 compares the result of the light quark asymmetry for the new ATLASepWZWjets 19 fit including the $p_{\mathrm{T}}(W)$, with the fit including the $W+$ jet leading jet $p_{\mathrm{T}}$. Also shown is the fit to the uncombined inclusive data only. For both fits including the $W+$ jet data the asymmetry is now mostly positive, each with a low maxima around $x=0.2$. This is more in line with the global fits which also see a larger $\bar{d}$ distribution than $\bar{u}$. The larger $\bar{d}$ distribution is one of the factors resulting 

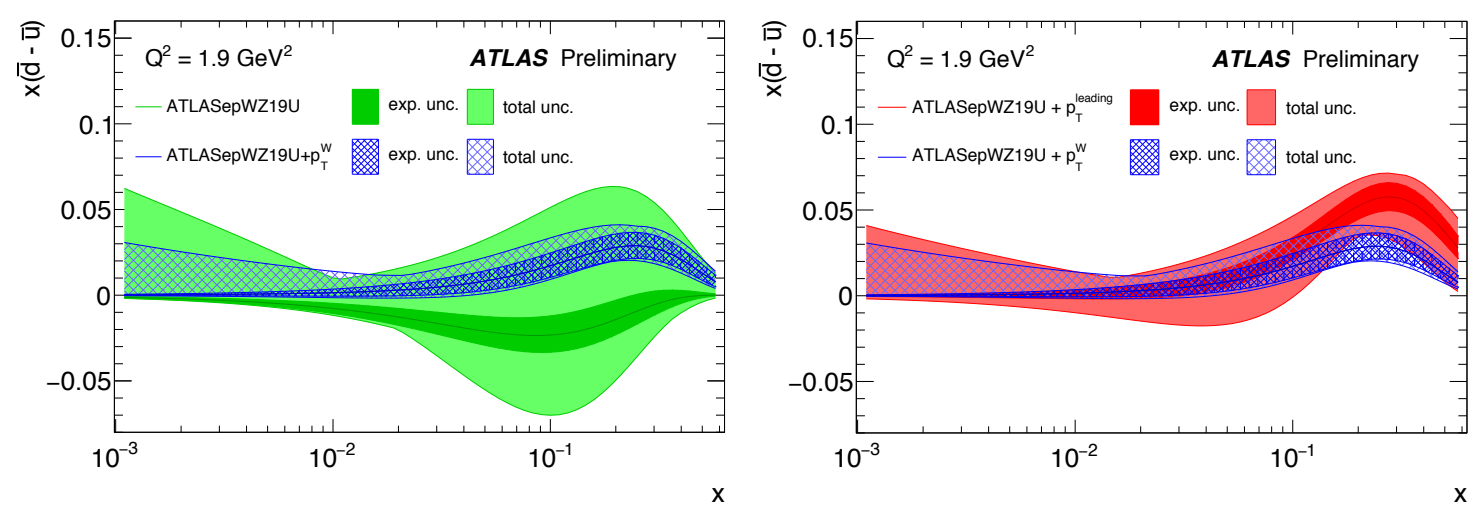

Figure 4: The light quark asymmetry, $x(\bar{d}-\bar{u})$, for the fits discussed in the text. The left figure compares the fit including only the inclusive $W$ and $Z$ data, to that obtained when also including the data for the $p_{\mathrm{T}}(W)$ distribution. The right figure compares the fits including the $p_{\mathrm{T}}(W)$, and the leading jet $p_{\mathrm{T}}$ distributions.

in the smaller value for the strangeness ratio at larger $x$. It should be noted that this positive light quark asymmetry is achieved while still maintaining the enhanced strangeness at low $x$, but is correlated with the reduction of the strangeness ratio at higher $x$.

A comparison with the results of the additional global fits from CT14 [17], MMHT2014 [18], NNPDF3.1 [19] and ABMP16 [20] for the value of $r_{s}$ evaluated at $x=0.023$ and $Q^{2}=1.9 \mathrm{GeV}^{2}$ can be seen in Figure 5. Also shown are values from the original ATLASepWZ16 fit and the new ATLASepWZ19U and ATLASepWZ19C fits to the inclusive $W$ and $Z$ data. The fits to the inclusive data are all in agreement, but predict a consistently higher value for the strangeness ratio than the new fit with the $W+$ jet data, as expected from the strangeness ratio discussed earlier. The new fit is more in line with the global fits, but still predicts an enhanced strangeness ratio. Comparing the value of the light quark asymmetry with the global fits, also in Figure 5, it is observed that the new asymmetry when including the $W+$ jet data is now more consistent with the observations from the global fits - albeit still predicting a slightly smaller ratio.

\section{Outlook}

ATLAS has an extensive, and growing portfolio of precision measurements, each having the potential to help constrain the parton distributions in the proton. For the analysis presented here a new fit including the inclusive $W$ and $Z$ data, enhanced by the inclusion of $W+$ jet data has been performed. The new fit confirms the enhancement of the strange contribution at low $x$, but now also with a positive light quark $x(\bar{d}-\bar{u})$ asymmetry, more consistent with the global fits.

There remain many analyses that could still benefit from the full luminosity of the Run I data. Together with the higher energy $13 \mathrm{TeV}$ collision data from LHC Run II, the potential to even more tightly constrain the proton PDF is great. Including more published data is possible - for example, data from top production or other vector boson data - and new precision vector boson data will be available soon, including new data on ATLAS $Z+$ jet production. New inclusive vector boson data at higher, and lower beam energies should lead to further reductions in both the statistical and systematic uncertainties, with better constraints on the gluon. For many measurements, the 

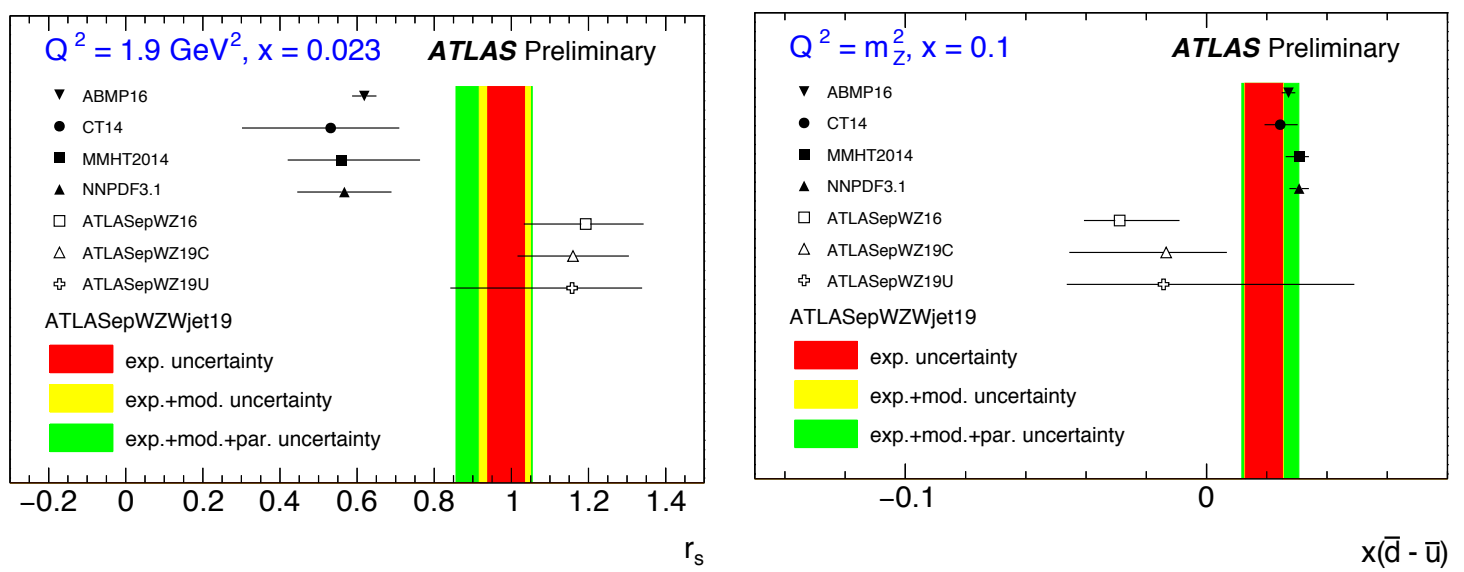

Figure 5: A summary of the strangeness ratio, $r_{s}$ evaluated at $Q^{2}=1.9 \mathrm{GeV}^{2}$ and $x=0.023$ (left) and the light quark asymmetry evaluated at the $Z$ mass and with $x=0.1$ (right). Results are shown comparing the values from the new fits with those from the four major global PDF sets.

theoretical uncertainties are often comparable to, or larger, than those from the data. New NNLO calculations for heavy boson production in association with a jet [21], are now available, and within the same framework, many other significant physics processes are also available. Developments in the interface of the calculations with the fast interpolation grid technology mean these data - and HERA jet data - will be usable in rigorous NNLO fits, including the inclusive $W$ and $Z$ production data, as well as that accompanied by a hadronic jet.

Although a great deal of progress has been achieved in the field of QCD fits including LHC data, the journey towards realising the full potential of these data is only just beginning, and it promises to be a very interesting time ahead.

\section{References}

[1] L. Evans and P. Bryant, JINST 3 (2008) S08001.

[2] ATLAS Collaboration, G. Aad et al., JINST 3 (2008) S08003.

[3] J. M. Campbell and R. K. Ellis, Nucl. Phys. Proc. Suppl. 10 (2010) 205-206, arXiv:1007. 3492.

[4] J. M. Campbell and R. K. Ellis, Phys. Rev. D60 (1999) 113006, arXiv: hep-ph/ 9905386.

[5] T. Carli, G. P. Salam and F. Siegert, "A Posteriori inclusion of PDFs in NLO QCD final-state calculations," in HERA and the LHC: A Workshop on the Implications of HERA for LHC Physics (Startup Meeting, CERN, 26-27 March 2004; Working Group Meeting, CERN, 17-21 January 2005; Final Meeting 21-24 Mar 2005) CERN, Geneva, Switzerland, October 11-13, 2004. 2005. arXiv:hep-ph/0510324.

[6] T. Carli et al., Eur. Phys. J. C66 (2010) 503, arXiv:0 911.2985.

[7] S. Catani and M. Grazzini, Phys. Rev. Lett. 98 (2007) 222002, arXiv: hep-ph/ 0703012.

[8] S. Catani, L. Cieri, G. Ferrera, D. de Florian and M. Grazzini, Phys. Rev. Lett. 103 (2009) 082001, arXiv:0903.2120. 
[9] R. Gavin, Y. Li, F. Petriello and S. Quackenbush, Comput. Phys. Commun. 182 (2011) 2388-2403, arXiv:1011.3540.

[10] R. Gavin, Y. Li, F. Petriello and S. Quackenbush, Comput. Phys. Commun. 184 (2013) 208-214, arXiv:1201.5896.

[11] ATLAS Collaboration, M. Aaboud et al., Eur. Phys. J. C77 (2017) 367, arXiv: 1612.03016.

[12] A. M. Cooper-Sarkar and K. Wichmann, Phys. Rev. D 98 (Jul, 2018) 014027, arXiv: 1803.00968.

[13] ATLAS Collaboration, "Determination of the parton distribution functions of the proton from ATLAS measurements of differential $W$ and $Z / \gamma^{*}$ and $t \bar{t}$ cross sections," ATL-PHYS-PUB-2018-017, CERN, Geneva, Aug 2018.

[14] ATLAS Collaboration, M. Aaboud et al., Phys. Rev. D94 (2016) 092003, arXiv: 1607.07281.

[15] ATLAS Collaboration, G. Aad et al., Eur. Phys. J. C76 (2016) 538, arXiv: 1511.04716.

[16] ATLAS Collaboration, M. Aaboud et al., JHEP 05 (2018) 077, arXiv:1711. 03296.

[17] S. Dulat, T.-J. Hou, J. Gao, M. Guzzi, J. Huston, P. Nadolsky, J. Pumplin, C. Schmidt, D. Stump and C. P. Yuan, Phys. Rev. D 93 (2016) 033006, arXiv:1506.07443.

[18] L. A. Harland-Lang, A. D. Martin, P. Motylinski and R. S. Thorne, Eur. Phys. J. C 75 (2015) 204, arXiv:1412.3989.

[19] NNPDF Collaboration, R. D. Ball et al., Eur. Phys. J. C 77 (2017) 663, arXiv: 1706.00428.

[20] S. Alekhin, J. Blümlein, S. Moch and R. Placakyte, Phys. Rev. D 96 (2017) 014011, arXiv:1701.05838.

[21] A. Gehrmann-De Ridder, T. Gehrmann, E. W. N. Glover, A. Huss and T. A. Morgan, Phys. Rev. Lett. 117 (2016) 022001, arXiv:1507.02850. 